\title{
OPINIÃO DAS GESTANTES SOBRE ACOMPANHAMENTO DA ENFERMEIRA OBSTETRA NO PRÉ-NATAL DE UM HOSPITAL UNIVERSITÁRIO
}

\author{
PREGNANT WOMEN'S OPINION ABOUT THE OBSTETRICS NURSE \\ SUPPORT DURING PRENATAL AT A UNIVERSITY HOSPITAL
}

\section{OPINIÓN DE LAS GESTANTES SOBRE EL ACOMPAÑAMIENTO DE LA ENFERMERA OBSTETRA EN EL PRENATAL DE UN HOSPITAL UNIVERSITARIO}

\author{
Thelma SPindola \\ Jane Márcia Progianti ${ }^{*}$ \\ Lucia Helena Garcia Penna ${ }^{* *}$
}

\begin{abstract}
RESUMO
Trata-se de um estudo qualitativo, exploratório, descritivo que foi realizado em um Hospital Universitário no município do Rio de Janeiro, com objetivo de descrever a percepção das gestantes em relação ao atendimento da enfermeira obstetra no acompanhamento do pré-natal. Após aprovação da pesquisa pelo Comitê de Ética da referida instituição, foram investigadas 18 gestantes que concordaram em responder uma entrevista semiestruturada. A análise temática dos relatos revelou que o atendimento da enfermeira é diferenciado, com acolhimento e atenção, facilitando o esclarecimento de dúvidas e o aprendizado em relação ao processo de gestar e parir. O vínculo estabelecido entre a enfermeira obstetra e a gestante no pré-natal contribui para o processo de educação para a saúde das mulheres, respeitando-se os hábitos de vida e a cultura da clientela.
\end{abstract}

Palavras chave: Cuidado pré-natal, Enfermagem obstétrica, gestação, saúde da mulher.

\begin{abstract}
This is a qualitative, exploratory and descriptive study carried out in a University Hospital located in the city of Rio de Janeiro, aiming to describe the pregnant women's perception regarding the obstetrics nurse care at prenatal support clinic. Eighteen pregnant women, who agreed to answer a partially-structured interview, were investigated after the research was approved by the institution's Ethics Committee. The thematic analysis of the accounts revealed that the obstetrics nurse care is differentiated, with warmth and attention, thus enabling the clarification of questions and learning in relation to the pregnancy and childbearing processes. The prenatal connection established between the obstetrics nurse and the pregnant woman contributes for the process of health education for women, respecting the clients' life habits and culture.
\end{abstract}

Key words: Prenatal care, Obstetrical nursing, pregnancy, woman's health.

\footnotetext{
* Doutora em Enfermagem. Professora adjunta da Faculdade de Enfermagem da Universidade do Estado do Rio de Janeiro. E-mail: spindola@predialnet.com.br

${ }^{* *}$ Doutora em Enfermagem. Professora adjunta da Faculdade de Enfermagem da Universidade do Estado do Rio de Janeiro. E-mail: jmprogi@uol.com.br

${ }^{* * *}$ Doutora em Enfermagem. Professora adjunta da Faculdade de Enfermagem da Universidade do Estado do Rio de Janeiro. E-mail: luciapenna@terra.com.br
} 


\section{RESUMEN}

Se trata de un estudio cualitativo, exploratorio, descriptivo que fue realizado en un hospital universitario en el municipio de Río de Janeiro, con el objetivo de describir la percepción de las gestantes en relación con la atención de la enfermera obstetra en el acompañamiento del prenatal. Posterior a la aprobación de la investigación por parte del Comité de Ética de la referida institución, fueron investigadas dieciocho embarazadas que accedieron a responder una entrevista semiestructurada. El análisis temático de los relatos reveló que la atención de la enfermera es diferenciada, con acogida y atención, facilitando la aclaración de las dudas y el aprendizaje en relación con el proceso de embarazo y parto. El vínculo establecido entre la enfermera obstetra y la gestante en el prenatal contribuye en el proceso de educación para la salud de las mujeres, respetándose los hábitos de vida y la cultura de la clientela.

Palabras clave: Atención prenatal, Enfermería obstétrica, embarazo, salud de la mujer.

Fecha recepción: 13/06/11 Fecha aceptación: 14/08/12

\section{INTRODUÇÃO}

A gestação é um fenômeno fisiológico e sua evolução acontece, na maioria dos casos, sem intercorrências. A atenção pré-natal e puerperal tem como principal objetivo o acolhimento da mulher desde o início da gravidez assegurando o nascimento de uma criança saudável e o bem-estar materno e neonatal (1). Entendida como um processo dinâmico e transformador a gestação apresenta dimensões sócio-culturais, além das modificações físicas evidenciadas no corpo da mulher. Os cuidados no pré-natal devem ultrapassar a questão biológica e tecnicista de atenção às gestantes e, a assistência deve propiciar um adequado andamento das gestações para que ocorra de forma saudável (2).

A enfermeira cuidando da gestante com a percepção da dinâmica que se estabelece entre a mulher e seu grupo social com a gravi$\mathrm{dez}$, tem instrumentos essenciais para o desenvolvimento de uma prática humanizada segundo as recomendações da Organização Mundial de Saúde (3). O cuidado humanizado no pré-natal é o primeiro passo para um nascimento saudável, diminuição da morbimortalidade materna e fetal, aquisição de autonomia e vivência segura no ciclo gravídico puerperal (4).
A consulta no pré-natal é um momento importante de interação entre a mulher gestante e o profissional de saúde sendo uma ocasião propícia para o esclarecimento de dúvidas, a troca de experiências e/ou conhecimentos e a compreensão do processo de gestar. Tem o objetivo de cuidar da mulher e seu filho no período gestacional, considerando o contexto familiar e social da gestante, preparando-a para um parto e puerpério seguros e saudáveis, sendo um espaço privilegiado para que a mulher se sinta segura e confiante para trazer seus questionamentos e possa discuti-los. A consulta de enfermagem é um espaço de acolhimento que possibilita o diálogo e permite verbalizar dúvidas, sentimentos, e experiências o que contribui para estreitar o vínculo entre a enfermeira e a gestante (5).

O enfermeiro deve compreender a importância de humanizar e qualificar a atenção à gestante, para obter maior adesão ao pré-natal, e garantir a qualidade na assistência com melhores resultados obstétricos e perinatais (6). A postura acolhedora e respeitosa dos profissionais e a escuta qualificada às expectativas e necessidades da mulher podem, portanto, ser um estímulo para adesão ao serviço de pré-natal (4).

Na rede básica de saúde do município do Rio de Janeiro a consulta de enfermagem 
no pré-natal é realizada conforme o roteiro estabelecido pelo Ministério da Saúde (1) e se apresenta como um instrumento importante que têm como finalidade garantir a extensão da cobertura e melhoria da qualidade pré-natal, principalmente por meio da introdução das ações de preventivas e promocionais às gestantes com baixo risco obstétrico (5).

No Hospital Universitário Gaffrée e Guinle, da Universidade Federal do Estado do Rio de Janeiro (HUGG-UNIRIO), a consulta de enfermagem no pré-natal foi instituída no ano de 2000. Anterior a este período ocorriam orientações coletivas para a saúde das gestantes sendo inseridas no atendimento do pré-natal do hospital, realizado exclusivamente por médicos. As mulheres eram matriculadas no hospital, recebiam a solicitação dos exames de rotina e tinham sua consulta agendada com o médico obstetra. A demanda de gestantes era superior a oferta de vagas e muitas mulheres aguardavam a primeira consulta no pré-natal por um período de 2 a 3 meses. Em 2000, foi estruturado um Projeto de Extensão denominado "Consulta de Enfermagem às gestantes do HUGG-UNIRIO" minimizando o tempo de espera de um atendimento especializado para a detecção precoce de possíveis intercorrências obstétricas.

Desde, então, todas as grávidas no HUGG passaram a ter seu primeiro contato na instituição com a equipe do Projeto, coordenado por uma enfermeira obstetra, incluindo residentes e alunos de graduação em enfermagem da Escola de Enfermagem Alfredo Pinto (EEAP-UNIRIO). É oportuno registrar que nosso ambiente de trabalho nem sempre foi harmonioso. No começo houve resistência por parte de alguns médicos obstetras que não aceitavam a participação da enfermeira obstetra no atendimento às gestantes, exceto nas atividades coletivas de orientações para a saúde. Em decorrência deste fato, e, também porque na ocasião o hospital era referência para gestações de risco, a consulta de enfer- magem se limitava, a princípio, ao primeiro atendimento da gestante com solicitação de exames e encaminhamento ao obstetra para acompanhamento no pré-natal.

Em 2003, a partir dos resultados de uma pesquisa que delimitou o perfil epidemiológico das mulheres atendidas no pré-natal do HUGG, pudemos constatar que aproximadamente $80 \%$ da população estudada era composta por mulheres classificadas como gestantes fisiológicas, ou seja, sem risco obstétrico. Esses achados justificaram a instituição da interconsulta de enfermagem como um campo de atuação da enfermeira obstetra junto às mulheres que apresentavam gestações fisiológicas. Neste campo a enfermeira obstetra realiza o primeiro atendimento, conforme o protocolo, e as consultas subsequentes intercaladas com a consulta médica. Se por um lado, a criação da interconsulta nos mostra que as ações da enfermeira obstetra ainda que, amparadas legalmente (1, 7), sofrem resistência da corporação médica, por outro lado, significou uma estratégia de luta das enfermeiras na ocupação deste espaço do campo hospitalar. No entanto, a manutenção deste espaço está vinculada às articulações políticas na dinâmica do campo, à adesão das mulheres e à qualidade do atendimento.

Cabe ressaltar, todavia, que a interconsulta não foi aceita por todos os médicos, que marcavam atendimento na mesma data da enfermeira obstetra e, por conseguinte, muitas mulheres solicitavam o re-agendamento da consulta com a enfermeira. Neste contexto esta investigação teve como questões norteadoras: Qual a percepção da gestante em relação à consulta de enfermagem no pré-natal do HUGG-UNIRIO? Como as mulheres avaliam o atendimento da enfermeira obstetra?

O estudo teve o objetivo de descrever a percepção das gestantes em relação ao atendimento da enfermeira obstetra no pré-natal do HUGG-UNIRIO. 


\section{MATERIAL E MÉTODO}

Trata-se de um estudo exploratório, descritivo, qualitativo que buscou analisar a consulta de enfermagem no pré-natal do HUGG-UNIRIO. O projeto foi analisado pelo Comitê de Ética em Pesquisa da instituição, sendo aprovado com o número 62/2004.

O cenário do estudo foi um hospital universitário federal localizado no município do Rio de Janeiro. Nesta instituição uma enfermeira obstetra, lotada no Serviço de Pacientes Externos, é responsável pela inserção e agendamento das mulheres grávidas no prénatal. Toda gestante para ser absorvida no pré-natal participa de uma atividade coletiva (grupo de gestantes) e, posteriormente, sua consulta é agendada com a enfermeira. As gestantes que apresentam o perfil de baixo risco (gestação fisiológica) são convidadas a participar da interconsulta, ou seja, participar do acompanhamento médico e da enfermeira durante o pré-natal. A coleta dos dados foi realizada no período de dezembro de 2004 a março de 2005 com o auxílio de duas residentes de enfermagem da EEAP-UNIRIO que colaboraram na coleta das informações e realização das entrevistas.

As gestantes que compareceram de 4 a 5 consultas de enfermagem e, na ocasião, estavam no $3^{\circ}$ trimestre gestacional (em torno de 37 semanas) foram convidadas a participar da investigação. Esclarecidas quanto aos objetivos do estudo, assinaram o Termo de Consentimento Livre e Esclarecido, respeitando-se os aspectos éticos para a realização de pesquisas com seres humanos. Responderam a uma entrevista semi-estruturada com questões relacionadas às características sóciodemográficas e obstétricas e 3 questões abertas: Como você percebeu a consulta com a enfermeira obstetra durante o pré-natal? Como você avalia o atendimento realizado pela enfermeira? Você se sentiu acolhida e com liberdade para expor seus receios e esclarecer suas dúvidas na consulta com a en- fermeira? As entrevistas ocorreram no horário de atendimento da consulta do pré-natal, sendo utilizado o recurso de gravação em fita magnética com a anuência das entrevistadas.

A coleta das entrevistas foi finalizada quando ocorreu a saturação das informações tendo totalizado 18 encontros. Procedemos à transcrição e análise das descrições com emprego da técnica de análise temática (8), que permite a leitura e interpretação do conteúdo de qualquer classe de documentos, auxiliando na análise para a compreensão de seus significados. Procedemos a pré-análise (seleção dos documentos importantes); exploração do material (tendo-se realizado a leitura e releitura do material transcrito nas entrevistas e definição das unidades de registro) e tratamento dos resultados, interferência e interpretação (identificação dos temas e categorias correlacionando-o ao referencial adotado) (8). Atribuímos um pseudônimo às mulheres para preservar o anonimato das participantes do estudo.

\section{RESULTADOS}

As mulheres entrevistadas tinham a seguinte caracterização: 14 tinham idades entre 19 e 24 anos e 4 de 25 a 30 anos de idade; quanto à escolaridade 10 tinha o ensino médio e 8 o ensino fundamental; quanto à ocupação 7 exerciam atividade remunerada e 11 não exerciam. A renda familiar das mulheres era até $R \$ 520,00$, ou seja, 1 a 2 salários (considerando que em 2004 o salário mínimo nacional era $\mathrm{R} \$ 260,00)$. Em relação ao número de gestações 7 eram primigestas e 11 secundigestas.

Os dados empíricos foram organizados e submetidos à leitura e releitura visando à obtenção dos núcleos de sentido, extraindose três categorias temáticas, a saber: A criação do vínculo como diferencial na consulta de enfermagem; $\mathrm{O}$ acolhimento e a atenção como atitude no cuidar; Liberdade 
de expressão e esclarecimento de dúvidas conseqüentes ao estabelecimento de relações menos assimétricas.

A análise das entrevistas com as gestantes revelou que na consulta de enfermagem existe pouco revezamento de profissionais o que favorece a aproximação entre a mulher e a enfermeira. A continuidade do profissional nas consultas ajuda, na opinião das mulheres, a aumentar sua confiança no atendimento e a estabelecer um vínculo:

"[...] na enfermagem pelo menos você conhece [a enfermeira] então, como você é consultada por duas, três vezes com a mesma pessoa, a gente pega mais confiança [...] porque com o doutor cada vez é um e você tem que falar tudo de novo, é chato" (Dália).

"[...] porque a enfermeira $Z$ é muito legal, muito calma, explica tudo pra gente. Gosto de ser atendida por ela, confio mais do que nos médicos que cada vez é um que nos atende, e é tudo muito rápido [...], da última vez com ele [médico] não levou 10 minutos" (Margarida).

"[...] porque é a primeira vez que sou atendida por enfermeira no pré-natal. Gostei muito. Ela trata você com carinho, olha nos teus olhos, te dá bronca quando precisa e presta atenção na gente, até quando estou triste ela sabe e conversa comigo [...], diferente dos médicos sempre com pressa, não te dá muito papo" (Angélica).

$\mathrm{Na}$ análise das entrevistas pudemos notar que algumas gestantes percebem o acolhimento da enfermeira através do atendimento atencioso e paciente:

"O atendimento de vocês... vocês dão uma atenção assim especial. Escutam o coração do bebê, $v \hat{e}$ a pressão, pesa e conversa com a gente [...] é bem diferente" (Margarida).

"Eu gostei muito [...] as enfermeiras atendem com atenção, olham nos olhos, falam com paciência [...] ficava mais a vontade para pergun- tar e tirar minhas dúvidas. Muito diferente dos médicos que às vezes só escutam o coração do neném, gostei mais" (Flor de Liz).

Outras relataram perceber o acolhimento pelo bom atendimento que tiveram, pelo diálogo estabelecido com a enfermeira durante a consulta e pela privacidade com que foram ouvidas:

"[...] eu fui acolhida em todas as consultas, pelo atendimento que eu tive e pelo diálogo entre eu e a enfermeira. As minhas dúvidas elas tiravam, perguntavam se tinha entendido tudo. Me dava um papel [folder] com tudo explicado" (Orquídea).

"[...] desde o momento que cheguei aqui sempre fui bem atendida, bem tratada, na hora de chamar as enfermeiras sempre dão atenção àquela paciente, elas não bota aquele monte para falar tudo de uma vez, sempre é uma por uma. E não fica aquele monte de gente para te examinar. Eu me senti muito bem" (Camélia).

As falas das entrevistadas denotam que as relações de poder entre a enfermeira e as gestantes são menos autoritárias. As mulheres verbalizaram, também, que se sentiam mais à vontade nas consultas no pré-natal com a enfermeira o que favoreceu o esclarecimento de dúvidas, conforme os recortes de relatos clarificam:

"Foi bom porque eu tive mais liberdade com as enfermeiras para esclarecer minhas dúvidas, até porque o atendimento é mais devagar [...]" (Amarilis).

"O esclarecimento das dúvidas eu acho que foi isso o melhor para mim. Todas as dúvidas que eu tive eu perguntei e tudo foi respondido. Até mesmo quando não era o dia da consulta ela (enfermeira) falava comigo e tirava dúvidas. Gostei muito daqui." (Petúnia).

"[...] eu me sinto assim mais segura, porque 
tiraram as minhas dúvidas, que com o médico eu to conversando com ele, e ele não tá nem aí, tá sempre saindo, então as enfermeiras não, elas têm um atendimento bem melhor, não têm pressa, dão atenção” (Angélica).

As mulheres entrevistadas apontam como diferencial na consulta de enfermagem no pré-natal a orientação e o bom atendimento como um cuidado:

"[...] do meu outro filho eu fiz pré-natal somente com o médico, não tinha enfermeira no pré-natal. Este agora foi muito melhor. Me sinto mais segura porque vocês explicam tudo direitinho. Examinam melhor, toda consulta me pesam, vê a pressão, mede a barriga, escuta o bebê [...] Eu não tive vergonha de perguntar [...] até quando não tinha dinheiro para vir para a consulta ou comprar o remédio que o médico passou eu falei para ela (enfermeira) e me encaminhou para a assistente social, nossa, foi muito legal!” (Azaleia).

\section{DISCUSSÃO E CONCLUSÃO}

As falas denotam confiança e reconhecimento do trabalho da enfermeira no atendimento às gestantes. $\mathrm{O}$ vínculo estabelecido entre as mulheres e a enfermeira obstetra pode ser observado nas falas de Margarida e Angélica. $O$ vínculo é um importante aliado para a permanência e adesão da mulher no atendimento do pré-natal, sendo um aspecto valorizado no Programa de Humanização do Parto e Nascimento (PHPN) (2, 9). Estudo que observou que entre os principais motivos de escolha do local para acompanhamento pré-natal apontado pelas gestantes está a experiência relatada por algum familiar já atendido naquele local, ter assegurado o parto no mesmo local de atendimento, empatia com profissionais da equipe (9). Para os autores embora o PHPN recomende a proximidade geográfica com a residência da mulher como único critério de referência para o local de realização do controle pré-natal, os fatores subjetivos são mais valorizados (9).

A unidade de saúde deve acolher a gestante e desenvolver cuidados com o objetivo de prevenir riscos e promover uma gravidez saudável (10). A adesão e a satisfação das mulheres com o atendimento no pré-natal estão relacionadas à qualidade da assistência prestada profissionais de saúde e pelos serviços $(2,10)$. Estudo constatou que a maioria das gestantes verbalizou satisfação com o acolhimento e atenção dos profissionais, enfatizando a importância do vínculo entre o profissional da Unidade Básica de Saúde e a gestante (10).

Neste sentido, autores (11) ressaltam a importância da participação dos enfermeiros para o fortalecimento da assistência pré-natal e a necessidade de investimentos na formação de pessoal qualificado para o atendimento à mulher no ciclo gravídico-puerperal. Avaliando a assistência pré-natal na perspectiva de puérperas e profissionais de saúde no município do Rio de Janeiro, observou-se que embora as puérperas tenham encontrado um atendimento diversificado em termos de especialidade, outros elementos também devem ser incorporados para proporcionar um ambiente mais acolhedor e alicerçado na humanização da assistência, sendo crucial neste processo a formação do vínculo para o maior envolvimento da gestante nas questões relacionadas à sua saúde (12).

Nas falas podemos notar que a comunicação e o acolhimento da enfermeira obstetra fizeram o diferencial no atendimento às mulheres. A comunicação, portanto, é um instrumento transformador e gerador de impacto à saúde e contribui na atividade de educação em saúde oferecendo atendimento à mulher em todas as dimensões de suas necessidades básicas (13). Os relatos deixam transparecer a relação de confiança estabelecida entre a mulher e a enfermeira, a empatia estabelecida entre ambas, denotando que as gestantes se sentiam acolhidas 
e cuidadas pela profissional. Considerando o pré-natal um momento ímpar torna-se um espaço adequado para a gestante preparar-se para viver o parto de forma positiva e integral. Neste cenário é imprescindível o estabelecimento de uma relação atenciosa pelos profissionais de saúde respeitando os limites de cada mulher, atentando para a sua cultura, seus hábitos de vida e, principalmente, criando um espaço de confiança para que ela possa expressar suas emoções, dúvidas e ansiedades.

$\mathrm{O}$ enfermeiro, na consulta de enfermagem, estabelece uma aproximação com o cliente, um vínculo de interação, de efetivo contato com o ser humano, possibilitando desvelar, descobrir dados sobre o estado de saúde ou de doença do cliente através da escuta atentiva e do cuidado (5). O acolhimento é um aspecto essencial da política de humanização do parto e nascimento e compreende a recepção da mulher na unidade de saúde, ouvindo suas queixas e permitindo que expresse suas preocupações e angústias. É uma ação que pressupõe a mudança de relação profissional/usuário sendo recomendada pelo Ministério da Saúde (1).

A enfermeira obstetra no HUGG estabelece o primeiro contato (acolhimento) com a gestante na atividade coletiva, ocasião que fazem o agendamento das consultas no pré-natal, sendo ou não incluídas no acompanhamento obstétrico da instituição. Àquelas que são incluídas, são assistidas no primeiro atendimento na consulta de enfermagem. As demais são encaminhadas para demais unidades de saúde, tendo-se a preocupação de escutar a história de cada mulher e proceder-se a orientação adequada.

A interação do enfermeiro com o cliente pode ser considerada um elemento essencial do cuidado, considerando que possibilita ao profissional conhecer as necessidades do cliente para melhor assisti-lo. A comunicação está presente em todas as ações da Enfermagem e exerce influência direta na qualidade da assistência prestada aos que necessitam de seus cuidados e na interação do enfermeiro com os clientes (14). Corroborando com este pensamento autores $(5,10)$ enfatizam que o diálogo é primordial para o cuidado humanizado, sendo necessário para que se superem as barreiras de comunicação existente entre os profissionais de saúde e as gestantes. Neste sentido, é imprescindível que a equipe interdisciplinar de saúde considere as crenças, cultura, valores e contexto de vida de mulheres em prol de un cuidado diferenciado (15).

As descrições das gestantes deixam transparecer que o diferencial no atendimento da enfermeira é a escuta atentiva, sem pressa, o esclarecimento das dúvidas, a confiança que permite a liberdade de expressão. No HUGG as gestantes buscam orientação com a enfermeira mesmo fora do dia aprazado para a sua consulta, conforme apontou Petúnia em seu relato. Elas percebem a disponibilidade da profissional, o estar-com, presente em todas as consultas, que propicia o entendimento e a empatia entre as mulheres e a enfermeira obstetra. Foi verbalizado por Angélica e Amarílis que o atendimento diferenciado da enfermeira é percebido pela escuta atentiva, pelas orientações e liberdade de expressão. A enfermeira no cuidado às mulheres procura deixá-las bem à vontade, permitindo e incentivando a presença de um familiar durante o atendimento, evita um quantitativo excessivo de profissionais (no máximo dois) e procura no atendimento subseqüente que a gestante seja atendida sempre pelo mesmo profissional para que se estabeleça um vínculo.

Autores (4) afirmam que a consulta prénatal na atenção básica brasileira, quase sempre, se caracteriza como um momento rotineiro, técnico, rápido e sem oportunidades para compartilhar conhecimentos e experiências, que cumpre protocolos institucionais onde se valorizam aferições e medidas. Esta realidade, também, pode ser apreendida no relato das gestantes ao descreverem as características da consulta com a enfermeira obs- 
tetra comparando com demais profissionais do pré-natal.

Em sua descrição, Azaleia afirma que o atendimento diferenciado da enfermeira obstetra permitiu que se sentisse segura e confiante para esclarecer suas dúvidas e revelar suas dificuldades financeiras sem constrangimento. Essa situação apontada pela depoente é comum nas instituições públicas de atendimento à saúde considerando o poder aquisitivo da clientela. Vale acrescentar que as mulheres participantes do estudo pertenciam a uma classe menos favorecida com baixo poder aquisitivo (renda mensal de 1-2 salários mínimos), o que muitas vezes inviabiliza a aquisição de medicamentos, e que o hospital não fornece medicamentos para pacientes atendidos no Serviço de Pacientes Externos.

Por outro lado, devemos considerar que a clientela além do baixo poder aquisitivo apresenta baixa escolaridade ( 8 entrevistadas tinham apenas o ensino fundamental), o que pode influenciar a compreensão dos procedimentos e/ou orientações para a sua saúde, sendo primordial que o profissional de saúde perceba a singularidade de cada mulher.

Entendendo a consulta de enfermagem como um momento de interação entre a gestante e a enfermeira, percebemos a importância deste encontro para favorecer $o$ contato da mulher com demais profissionais de saúde vivenciando o atendimento interdisciplinar, criando-se possibilidades para que suas necessidades sejam contempladas, considerando a diversidade do contexto econômico e sócio-cultural em que estão inseridas. Assim, concordamos com autores (5) ao afirmarem que a singularidade da consulta de enfermagem no pré-natal está atrelada à forma como se estabelecem as relações de comunicação da enfermeira com as gestantes, com valorização do acolhimento e da escuta atentiva superando, em parte, uma prática profissional centrada no modelo biológico. $\mathrm{O}$ bom atendimento profissional favorece $\mathrm{o}$ vínculo da usuária ao serviço de saúde, e as gestantes avaliam o desempenho do profissional pelo interesse demonstrado durante o exame físico, nas perguntas e orientações fornecidas e na resolutividade das condutas (6).

Podemos concluir que a consulta de enfermagem no pré-natal do HUGG/UNI-RIO tem contribuído no acompanhamento das gestantes fisiológicas e introduzido a abordagem multidisciplinar do cuidado à mulher grávida. Os encontros com as gestantes são essenciais para o estabelecimento de uma relação de confiança, para o esclarecimento de dúvidas e aproximação da mulher e seus familiares no processo de gestar.

$\mathrm{O}$ atendimento humanizado da enfermeira, respeitando o tempo de cada mulher, facilita o esclarecimento de dúvidas e o aprendizado em relação ao processo de gestar e parir. $O$ diferencial no atendimento da enfermeira obstetra está no acolhimento das mulheres respeitando os direitos da gestante como cidadã e auxiliando no processo de educação em saúde, valorizando a escuta atentiva. Os desafios no atendimento da enfermeira obstetra no pré-natal do HUGG não foram superados em sua totalidade, muito ainda temos que avançar, tendo-se ultrapassado apenas algumas etapas do processo de construção. Esta investigação possibilitou uma reflexão acerca do alcance das ações da enfermeira obstetra no acompanhamento do pré-natal fisiológico e a necessidade de ampliação do número de profissionais especializadas para atender a demanda com foco na qualidade da assistência.

Cabe ressaltar, todavia, que embora os dados tenham sido captados no HUGG em 2005, a percepção de gestantes em relação ao pré-natal realizado por enfermeiras é um tema atual, descrito na literatura e ratificado por mulheres assistidas na consulta com a enfermeira obstetra. Consideramos a temática relevante e acreditamos que seria oportuno que o estudo fosse replicado em outros 
cenários, multimetodológico, com diversidade de participantes para captar outras perspectivas do objeto em estudo.

\section{REFERÊNCIAS}

1. Brasil. Ministério da Saúde. Pré-natal e Puerpério Atenção Qualificada e Humanizada - manual técnico. Brasília; 2006.

2. Grando T, Cardoso SMM, Fontana R, Rodrigues FCP. Consulta pré-natal: satisfação das usuárias do SUS. Cogitare Enferm. 2012; 17(2): 336-41.

3. Camacho KG, Vargens OMC, Progianti JM, Spindola T. Vivenciando repercussões e transformações de uma gestação: perspectivas de gestantes. Cienc enferm. 2010; XVI(2): 115-25.

4. Zampieri MFM, Erdmann AL. Cuidado humanizado no pré-natal: um olhar para além das divergências e convergências. Rev Bras Saude Matern Infant. 2010; 10(3): 359-67.

5. Shimizu HE, Lima MG. As dimensões do cuidado pré-natal na consulta de enfermagem. Rev Bras Enferm. 2009; 62 (3): 387-92.

6. Barbosa TLA, Gomes LMX, Dias OV. O pré-natal realizado pelo enfermeiro: a satisfação das gestantes. Cogitare Enferm. 2011; 16(1): 29-35.

7. Conselho Federal de Enfermagem Brasil. Decreto 94406/87. [citado 15 julho 2010]. Disponível em: http//www.portalcofen. gov.br/node/4173

8. Bardin L. Análise de conteúdo. Lisboa: Edições 70; 2007.

9. Almeida CAL, Tanaka OY. Perspectivas das mulheres na avaliação do Programa de Humanização do Pré-natal e Nascimento. Rev Saude Pública 2009; 43(1): 98-104.

10. Silva RM, Costa MS, Matsue RY, Sousa GS, Catrib AMF, Vieira LJES. Cartografia do cuidado na saúde da gestante. Cienc Saude Colet. 2012; 17(3): 635-42.

11. Cunha MA, Mamede MV, Dotto LMG, Mamede FV. Assistência pré-natal: competências essenciais desempenhadas por enfermeiros. Esc Anna Nery. 2009; 13 (1): 145-53.

12. Líbera BD, Saunders C, Santos MMAS, Rimes KA, Brito FRSS, Baião MR. Avaliação da assistência pré-natal na perspectiva de puérperas e profissionais de saúde. Cienc Saude Colet. 2011; 16(12): 4855-64.

13. Souza VB, Roecker S, Marcon SS. Ações educativas durante a assistência prénatal: percepção de gestantes atendidas na rede básica de Maringá-PR. Rev Eletr Enf. [Internet]. $2011 \mathrm{abr} /$ jun [citado 8 agosto 2012]; 13(2): 199-210. Disponível em: http://www.fen.ufg.br/revista/v13/ n2/v13n2a06.htm.

14. Silva KL, Cruz DSM, Oliveira ICC, Nóbrega MML. Interação - instrumentos básicos do processo de cuidar em enfermagem. En: Nóbrega MML, Silva KL, organizadoras. Fundamentos do cuidar em enfermagem. 2a ed. Belo Horizonte: Aben; 2008/2009. p. 97-116.

15. Diaz CMG, Hoffmann IC, Costenaro RGS, Soares RS, Silva BR, Lavall BC. Vivências educativas da equipe de saúde em unidade gineco-obstétrica. Cogitare Enferm. 2010; 15(2): 364-7. 